\title{
Low energy constants from the chirally improved Dirac operator $D_{\mathrm{CI}}{ }^{*}$
}

\author{
Christof Gattringer \\ Karl-Franzens-Universität Graz, Austria \\ E-mail: christof.gattringer@uni-graz.at \\ Philipp Huber ${ }^{\dagger+}$ \\ Karl-Franzens-Universität Graz, Austria \\ E-mail: philipp.huber@stud.uni-graz.at \\ C. B. Lang \\ Karl-Franzens-Universität Graz, Austria \\ E-mail: christian.lang@uni-graz.at
}

The leading order low energy parameters like the pion decay constant or the quark condensate are well-known from "classical" low energy theorems and experiments. It is a challenge, however, to find these parameters based exclusively on an ab-initio QCD calculation. We discuss results of a quenched lattice calculation of low energy constants using the chirally improved Dirac operator. Several lattice sizes at different lattice spacings are studied, using pseudoscalar and axial vector correlators. We find consistent results for $f_{\pi}=96(2) \mathrm{MeV}, f_{K}=105(2) \mathrm{MeV}$, $\Sigma=-(286(4) \mathrm{MeV})^{3}$, the average light quark mass $\bar{m}=4.1(2.4) \mathrm{MeV}$ and $m_{s}=101(8) \mathrm{MeV}$.

XXIIIrd International Symposium on Lattice Field Theory

25-30 July 2005

Trinity College, Dublin, Ireland

\footnotetext{
*For the Bern-Graz-Regensburg (BGR) Collaboration.

$\dagger$ Speaker.

${ }^{\ddagger}$ Supported by Fonds zur Förderung der wissenschaftlichen Forschung in Österreich, project P16823-N08.
} 


\section{Setup}

When QCD was accepted as the quantum field theory of strong intereractions it became clear that its (approximate) chiral flavor symmetry is spontaneously broken. The exploitation of the underlying principles has then led to the development of chiral perturbation theory. In that context a systematic expansion of many observables in terms of low energy constants has been derived. These constants, however, have to be determined either from experiment or from basic principles, i.e., non-perturbative solution of the underlying field theory QCD. The lattice formulation of QCD allows such a determination.

Since chiral symmetry and its spontaneous and explicit breaking play an essential role, it is desirable to work with fermionic actions having that symmetry. Lattice Dirac operators obeying the Ginsparg-Wilson relation (GWR) are the lattice analogue of chirally symmetric continuum Dirac operators. Here we use the chirally improved Dirac operator $D_{\mathrm{CI}}$ [1]. It is based on a systematic expansion of the lattice Dirac operator taking into account the whole Clifford algebra and terms coupling fermions within a certain range of neighbors on the lattice. The expanded Dirac operator is inserted in the GWR which then leads to a set of algebraic equations for the expansion coefficients. In recent applications [2], as well as here, we use a set of 19 independent terms in the action. Finally, in the definition of $D_{\mathrm{CI}}$ also one step of HYP-smearing of the gauge configuration is included. For the gauge fields we use the Lüscher-Weisz action. The lattice spacings have been determined using the Sommer parameter. We summarize the simulation parameters in Table 1

In Ref. [2] hadron masses for a quenched simulation based on $D_{\mathrm{CI}}$ have been presented. Preliminary results for low energy constants in that context have been published in Ref. [3]. At that time the renormalization constants relating these to the continuum $\overline{\mathrm{MS}}$-scheme were not available. Meanwhile the necessary constants for quark bilinears have been determined for $D_{\mathrm{CI}}$ in [4]. This now allows us to compute some of the basic low energy parameters in the quenched case. For this purpose we study quenched QCD at various values of the quark masses and determine results for $m_{u}=m_{d} \neq m_{s}$ in the $u \bar{d}$ and $u \bar{s}$ meson sector for several lattice sizes and lattice spacings, down to a pion mass of $330 \mathrm{MeV}$.

All our results have been derived from correlation functions of pseudoscalar interpolating

\begin{tabular}{rcccccr}
$L^{3} \times T$ & $\beta$ & $a[\mathrm{fm}]$ & $a\left[\mathrm{GeV}^{-1}\right]$ & \#cf. & Type & $a m\left(a m_{s}\right)$ \\
\hline $8^{3} \times 24$ & 7.90 & 0.148 & 0.750 & 200 & $p, n$ & $0.02-0.20$ \\
$12^{3} \times 24$ & 7.90 & 0.148 & 0.750 & 100 & $p, n$ & $0.02-0.20$ \\
$12^{3} \times 24$ & 8.35 & 0.102 & 0.517 & 100 & $p, n$ & $0.02-0.20$ \\
$16^{3} \times 32$ & 7.90 & 0.148 & 0.750 & 99 & $p, n$ & $0.02-0.20$ \\
$16^{3} \times 32$ & 8.35 & 0.102 & 0.517 & 100 & $p, n$ & $0.02-0.20$ \\
$16^{3} \times 32$ & 8.70 & 0.078 & 0.395 & 100 & $p, n$ & $0.02-0.20$ \\
$16^{3} \times 32$ & 7.90 & 0.148 & 0.750 & 100 & $p, n, w$ & $0.02-0.20(0.08,0.10)$ \\
$20^{3} \times 32$ & 8.15 & 0.119 & 0.605 & 100 & $p, n, w$ & $0.017-0.16(0.06)$
\end{tabular}

Table 1: Parameters of the simulation. Where the strange quark mass is given (in parenthesis), we also determined propagators for strange hadrons. Type denotes the type of the quark source/sink and \# cf. the number of configurations entering the analysis. 


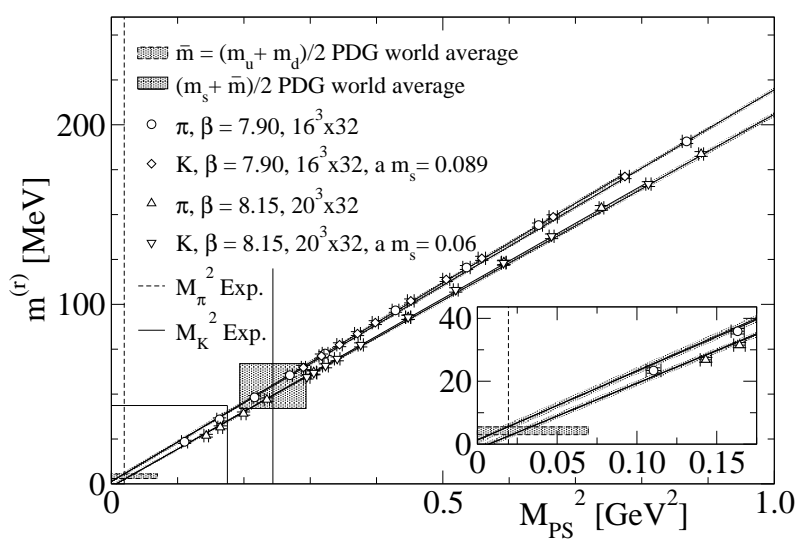

Figure 1: $m^{(r)}$ vs. $M_{P S}^{2}$ using $X=P$. Here $M_{P S}$ denotes $M_{\pi}$ or $M_{K}$, respectively. All masses are given in physical units in the $\overline{\mathrm{MS}}$-scheme.

fields $P=\bar{d} \gamma_{5} u$ and $A_{4}=\bar{d} \gamma_{4} \gamma_{5} u$. Where we give physical, renormalized ( $\overline{\mathrm{MS}}$-scheme) values, we always use the renormalization factors as obtained in the chiral limit, even in plots for nonvanishing bare quark masses. The renormalization factors $Z$ relate to the continuum $\overline{\mathrm{MS}}$-scheme at a scale of $\mu=2 \mathrm{GeV}$ and are $a$-dependent as given in [由. [7. We use the interpolating fields $P$ and $A_{4}$ for both the pion (with degenerate light quark mass $\bar{m}$ ), and the kaon, with one light and one strange quark. The bare mass of the heavy quark is fixed with the help of the physical kaon mass. In order to improve signals we use Jacobi-smeared sources and sinks for the quarks.

\section{Results}

In Ref. [5] we discuss the normalization of the sources, methods for fitting the propagators, error estimates (based on a jack-knife analysis), numerical derivatives, and the results for the meson masses. Here we present only some results for quark masses, condensate and the pseudoscalar decay constants.

\subsection{Quark masses}

The renormalized quark mass may be determined, utilizing the axial Ward identity,

$$
\frac{Z_{A}}{Z_{P}} \frac{\left\langle\partial_{t} A_{4}(\vec{p}=\overrightarrow{0}, t) X(0)\right\rangle}{\langle P(\vec{p}=\overrightarrow{0}, t) X(0)\rangle} \sim \frac{M_{\pi}^{2} f_{\pi}}{G_{\pi}^{(r)}}=Z_{m} 2 m=2 m^{(r)},
$$

where $X$ may be $P$ or $A_{4}$. The asymptotic behavior of the correlators cancels in this ratio and the plateau values provide the mass (proportional to the so-called AWI-mass). For the second choice, $X=A_{4}$, the correlators are of sinh-type and the ratio becomes numerically unstable near the symmetry point in $t$. Our analysis of the quark masses is based on the more stable choice $X=P$.

The quark mass data in Fig. 1, presented in physical units in the $\overline{\mathrm{MS}}$-scheme, leads to a very consistent picture. The abscissa gives the corresponding pseudoscalar mass, i.e., that of the pion or the kaon, for the corresponding values of $m^{(r)}$. We find that for given lattice spacing the numbers for kaon and pion are on top of each other, although these states have different quark content. 

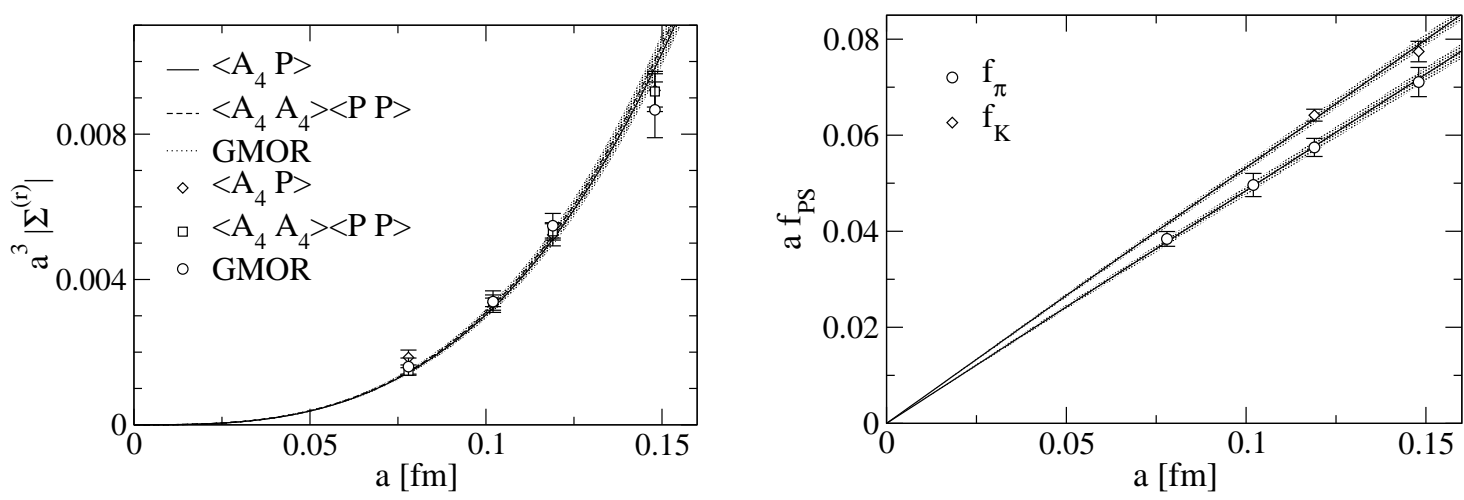

Figure 2: Scaling properties of the light quark condensate (1.h.s.) and of the pion decay constant (r.h.s).

The lines in Fig. 1 correspond to linear fits $m^{(r)} \propto M_{\pi}^{2}$, enforcing the simultaneous chiral limit of both observables. The data do not show deviation from that behavior, although logarithmic corrections are expected due to quenching. The linear extrapolations are in good agreement with the Particle Data Group [6] average for the light quark and strange masses at the physical pion mass. We obtain

$$
\frac{1}{2}\left(m_{u}^{(r)}+m_{d}^{(r)}\right) \equiv-{ }^{(r)} \simeq 4.1(2.4) \mathrm{MeV}, \quad \frac{1}{2}\left(m_{s}^{(r)}+{ }^{(r)}\right) \simeq 52(3) \mathrm{MeV}
$$

in the $\overline{\mathrm{MS}}$-scheme. Combing theses values gives $m_{s}^{(r)}=101(8) \mathrm{MeV}$. Possible finite size effects and other systematic effects like chiral extrapolation and quenching have not been accounted for. The given error takes into account the standard error and includes the derivations due to the dependence on the lattice spacing. The numbers are in good agreement with determinations from the overlap action in [7, §].

\subsection{Chiral condensate}

We have computed the renormalized condensate from the relations

$$
\begin{aligned}
f_{\pi}^{2} M_{\pi}^{2} & =-2 m^{(r)} \Sigma^{(r)}, \\
Z_{A} Z_{P}\left\langle A_{4}(\vec{p}=\overrightarrow{0}, t) P(0)\right\rangle \sim G_{\pi}^{(r)} f_{\pi} \mathrm{e}^{-M_{\pi} t}=\frac{f_{\pi}^{2} M_{\pi}^{2}}{2 m^{(r)}} \mathrm{e}^{-M_{\pi} t} & =\left|\Sigma^{(r)}\right| \mathrm{e}^{-M_{\pi} t}, \\
Z_{A} Z_{P} \sqrt{\left\langle A_{4}(\vec{p}=\overrightarrow{0}, t) A_{4}(0)\right\rangle\langle P(\vec{p}=\overrightarrow{0}, t) P(0)\rangle} & \sim\left|\Sigma^{(r)}\right| \mathrm{e}^{-M_{\pi} t},
\end{aligned}
$$

which all contain $\Sigma^{(r)}$. The first of these is the GMOR relation, the other two are determinations directly from the coefficients of propagators and implicitly related to GMOR as well.

We find excellent agreement for all three determinations; the values are consistent within the error bars. The dependence on the bare quark mass is compatible with the leading (linear) chiral behavior. Note, that we are not at small enough quark masses to be in the so-called $\varepsilon$-regime [9] but are in the $p$-regime.

In Fig. 2 (1.h.s) we show the results of the linear extrapolation to the chiral limit for all three types of determination for all lattice sizes studied. The expected scaling behavior $\mathscr{O}\left(a^{3}\right)$ is demonstrated by the fitted curves. Assuming the leading scaling behavior throughout (as was done in 


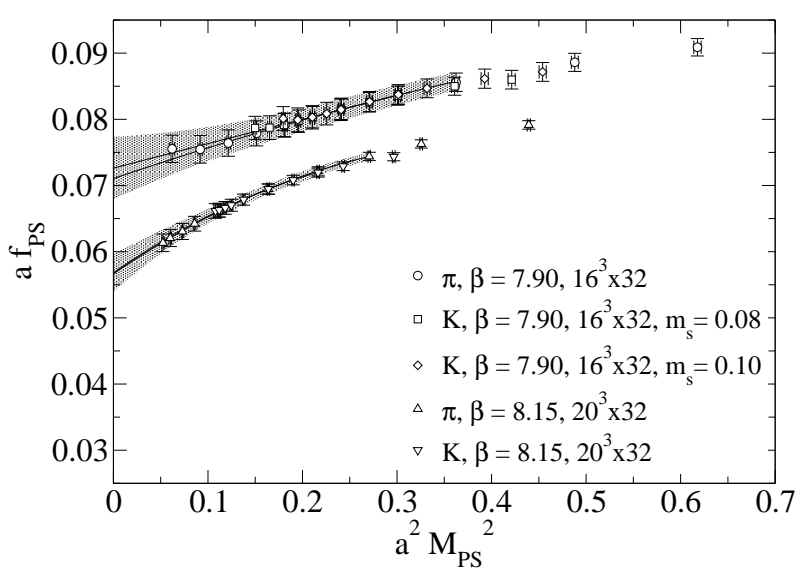

Figure 3: $a f_{\pi, K}$ vs. $\left(a M_{P S}\right)^{2}$ with error bands for chiral extrapolations as discussed in the text. The fit includes only data in the indicated range.

other studies where only one lattice spacing was studied) the average of the resulting values for the condensate in the continuum limit is $\left|\Sigma^{(r)}\right|=(286(4) \mathrm{MeV})^{3}$. This is slightly larger than a determination from the overlap action [12, 7] (although still with in the error limits) and larger than the corresponding results in Ref. [8, 13]. Our numbers are in good agreement with calculations in the $\varepsilon$-regime [10, 11].

\subsection{Decay Constants}

The pseudoscalar decay constants have been extracted from the asymptotic behavior of the pseudoscalar correlation functions according to

$$
Z_{A}^{2}\left\langle A_{4}(\vec{p}=\overrightarrow{0}, t) A_{4}(0)\right\rangle \sim M_{P S} f_{P S}^{2} \mathrm{e}^{-M_{P S} t}
$$

for pion and kaon, respectively. When plotting them as functions of the respective pseudoscalar masses in Fig. 3, the data for pion and kaon essentially overlap each other and exhibit a universal functional behavior. We also show the error band of a quadratic extrapolation to the chiral limit. In quenched QCD one expects correction terms with a logarithmic singularity in the valence quark mass $m$. As pointed out in [14], the leading order logarithmic term $m \log m$ of ChPT involves quark loops that are absent in the quenched case. There will be non-leading, e.g., logarithmic, terms, though. In addition to the term linear in the quark mass $m$ we therefore also allow a term $m^{2} \log m$ in the extrapolating fit (cf. the discussion in [15]). Actually, in the fit it makes no significant difference whether we take this term or just $m^{2}$.

In Fig. 2 (r.h.s.) we show the scaling behavior by comparing results for different lattice constants for our largest physical size lattices. The results are from the chiral extrapolation for the light quarks; in the (semi-) chiral extrapolation for the kaon decay constant the strange quark mass parameter is held fixed. Assuming the leading $\mathscr{O}(a)$ behavior, the continuum limit values are

$$
f_{\pi}=96(2) \mathrm{MeV}, \quad f_{K}=105(2) \mathrm{MeV} .
$$

Studies for the overlap action in quenched simulations have obtained similar results for $f_{\pi}[15,10$, 11, 13, 16, 17]. The experimental values are $f_{\pi}=92.4(0.3) \mathrm{MeV}$ and $f_{K}=113.0(1.3) \mathrm{MeV}$ [6]. 


\section{References}

[1] C. Gattringer, A new approach to Ginsparg-Wilson fermions, Phys. Rev. D 63 (2001) 114501 [hep-lat/ 0003005$]$; C. Gattringer, I. Hip, and C. B. Lang, Approximate Ginsparg-Wilson fermions: A first test, Nucl. Phys. B 597 (2001) 451 [hep-lat/ 0007042 ].

[2] C. Gattringer, M. Göckeler, P. Hasenfratz, et. al., Quenched spectroscopy with fixed-point and chirally improved fermions, Nucl. Phys. B 677 (2004) 3 [hep-lat/0307013].

[3] C. Gattringer et al., Quenched QCD with fixed-point and chirally improved fermion, Nucl. Phys. B (Proc. Suppl.) 119 (2003) 796 [hep-lat/0209099].

[4] C. Gattringer, M. Göckeler, P. Huber, and C. B. Lang, Renormalization of bilinear quark operators for the chirally improved lattice Dirac operator, Nucl. Phys. B 694179 (2004) 179 [hep-lat/0404006].

[5] C. Gattringer, P. Huber, and C. B. Lang, Lattice calculation of low energy constants with Ginsparg-Wilson type fermions, [hep-lat/0509003].

[6] S. Eidelman et al., Review of particle physics, Phys. Lett. B 592 (2004) 1.

[7] L. Giusti, C. Hoelbling, and C. Rebbi, Quenched results for light quark physics with overlap fermions, Nucl. Phys. B (Proc. Suppl.) 106 (2002) 739 [hep-lat/0110184]; R. Babich et al., Light hadron and diquark spectroscopy in quenched QCD with overlap quarks on a large lattice, [hep-lat/0509027].

[8] T.-W. Chiu and T.-H. Hsieh, Light quark masses, chiral condensate and quark-gluon condensate in quenched lattice QCD with exact chiral symmetry, Nucl. Phys. B673 (2003) 217 [hep-lat/0305016].

[9] P. Hernandez, K. Jansen, and L. Lellouch, Finite-size scaling of the quark condensate in quenched lattice QCD, Phys. Lett. B 469 (1999) 198 [hep-lat/9907022].

[10] L. Giusti, P. Hernandez, M. Laine, P. Weisz, and H. Wittig, Low-energy couplings of QCD from current correlators near the chiral limit, JHEP 0404 (2004) 013 [hep-lat / 0402002 ].

[11] H. Fukaya, S. Hashimoto, and K. Ogawa, Low-lying mode contribution to the quenched meson correlators in the epsilon-regime, [hep-lat/0504018].

[12] J. Wennekers and H. Wittig, On the renormalized scalar density in quenched QCD, [hep-lat/0507026].

[13] W. Bietenholz and S. Shcheredin, Overlap hypercube fermions in QCD with light quarks, PoS (LAT2005) 138 (2005) [hep-lat/0508016]; Low energy constants from the zero mode contribution to the pseudo-scalar correlator, ibid. 134 (2005) [hep-lat/0508034].

[14] S. R. Sharpe, Quenched chiral logarithms, Phys. Rev. D 46 (1992) 3146 [hep-lat/9205020].

[15] S. J. Dong et al., Chiral logs in quenched QCD, Phys. Rev. D 70 (2004) 034502 [hep-lat/0304005].

[16] S. Dürr and C. Hoelbling, Continuum physics with overlap fermions, [hep-ph/0508085].

[17] P. H. Damgaard, U. M. Heller, K. Splittorff, and B. Svetitsky, A new method for determining $F(p i)$ on the lattice, [hep-lat/0508029]. 